\title{
Memory, market stability and attractors coexistence in a nonlinear cobweb model
}

\section{Ahmad Naimzada \& Nicolò Pecora}

To cite this article: Ahmad Naimzada \& Nicolò Pecora (2016): Memory, market stability and attractors coexistence in a nonlinear cobweb model, Journal of Difference Equations and Applications, DOI: 10.1080/10236198.2016.1142540

To link to this article: http://dx.doi.org/10.1080/10236198.2016.1142540

\section{Published online: 10 Feb 2016.}

\section{Submit your article to this journal $₫$}

Llll Article views: 36

Q View related articles ¿

View Crossmark data ¿ 


\title{
Memory, market stability and attractors coexistence in a nonlinear cobweb model
}

\author{
Ahmad Naimzada ${ }^{a}$ and Nicolò Pecora ${ }^{b}$

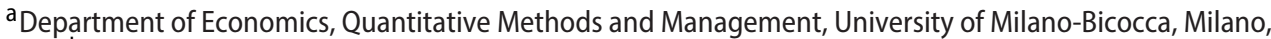 \\ Italy; ${ }^{b}$ Department of Economics and Social Science, Catholic University, Piacenza, Italy
}

\begin{abstract}
We investigate the dynamics of a cobweb type model with nonlinear demand and supply curves in which producers make forecasts on future prices with a backward looking expectation formation mechanism: the expected price for the next period is obtained by a weighted average of the prices observed in the last two periods. The study herewith presents aims at confirming the existence of a locally stabilising effect due to the presence of memory, but an increase of memory in price expectations can be globally qualitatively destabilising, in the sense that it leads to coexistence of different attractors with their respective basins of attraction.
\end{abstract}

\section{ARTICLE HISTORY}

Received 7 April 2015

Accepted 10 January 2016

\section{KEYWORDS}

Cobweb model; memory; stability; bifurcation analysis; attractors coexistence; Arnold tongues

\section{Introduction}

The cobweb model is a classical partial equilibrium framework to describe commodity price fluctuations of a non-storable goods (such as corn or hogs) as a result of the interaction between demand and supply, and that takes one time period to produce. In the first half of the past century Ezekiel's seminal article [9] proposed a model with linear supply and demand and static expectations. Ezekiel drew attention to the fact that whether stability or instability arises in the naive predictive model is dependent on the values of parameters representing the demand and supply reactivities. A rich literature has explored and extended the original formulation. Just to mention few, in [21] Nerlove introduced adaptive expectations in the traditional model and discovered a stabilising effect on the system; Manning extended the model by providing the conditions for the fixed point stability when nonlinear functions are considered [20]; along the lines of Carlson [6], authors in [20] and [11] have shown that, in a nonlinear model, equilibrium is stable if agents use the mean value of past prices to make forecasts. Even though all of these contributions added more realistic elements to the native framework, the dynamics remained simple. Subsequently, the hypothesis of nonlinear yet monotonic supply associated to adaptive expectations became the object of research for both Chiarella (see [7]) and Hommes (see [12]). Their works confirmed that when agents learn from the past they contribute to stabilise the market but the existence of nonlinearity may lead to chaotic behaviour. More recently authors in [8] considered a cobweb model with two

CONTACT Nicolò Pecora nicolo.pecora@unicatt.it 
interacting markets where producers have the choice between the two markets. Such an interaction destabilises otherwise stable markets and generate complex dynamics.

On the basis of the results already obtained, we study a model on price dynamics in a more realistic commodity market, in which both demand and supply functions are nonlinear and where agents use a memory learning mechanism to form expectations. In particular, differently from [13] and [14], which consider a linear demand curve and an Sshaped, increasing nonlinear supply curve, we consider a nonlinear demand function and a nonlinear convex supply function. Producers form expectations with a backward looking expectation formation mechanism: the expected price for the next period is obtained by a weighted average of the prices observed in the last two periods. A similar expectation formation mechanism has also been proposed in a different macroeconomic framework in [5]. Memory can have a smoothing effect on the price dynamics, leading to reduced or no amplitudes in price fluctuations. The adopted assumptions give rise to a 2-dimensional map which includes a behavioural parameter representing the degree of memory of the producers while formulating their price expectations.

The analysis of this dynamic system reveals that memory has a stabilising role for a weighted average close to a uniform distribution; otherwise, when the memory parameter takes extreme values (i.e. when consideration of the last price realisation prevails or it is too much neglected), the system can be destabilised through period doubling or NeimarkSacker bifurcation. Through the stability analysis we analytically prove the occurrence of the local bifurcations that lead to stability loss of the unique equilibrium, and the boundary of the stability region in the space of parameters is obtained. Furthermore, a numerical inquiry of the global dynamics of the model clearly shows that further dynamic behaviours have to be considered, characterised by coexistence of attracting sets, each with its own basin of attraction and even in the range of stability of the unique steady state. The eventuality of coexistence of the locally stable equilibrium with periodic attractors characterizing different kind of long-run dynamics, is shown numerically, as well as the structure of the basins of attraction that characterises the path dependance property of the cobweb model with memory. This fact deserves mention because it gives an alternative between two points of view, one stating that any perturbation from the steady state is recovered by the endogenous dynamics that goes back to the equilibrium in the long run, and the other referring to the fact that any small perturbation from the equilibrium is amplified by the endogenous dynamics, leading to a different attractor characterised by self sustained oscillations. In the case of coexistence, a situation known in the economic literature as corridor stability (see [19]) is obtained, that is small perturbations are recovered as far as they are confined inside the basin of attraction of the locally stable steady state, whereas longer perturbations lead to time evolutions that further depart from the equilibrium and go to the coexisting attractor in the long run, where oscillatory motion prevails. Besides such a phenomenon of coexistence, we find a rich periodicity structure through the presence in the parameter space of Arnold tongues, that is periodicity regions related to the existence of cycles organised according to the Farey summation rule applied to the rotation number of the related cycles (see [3]).

The paper is organised as follows: in Section 2 we briefly describe the cobweb model; in Section 3 we introduce backward looking expectations and we provide analytical results either in the case of no memory or in the presence of memory, showing how such a parameter affects the local stability of the unique steady state. In Section 4 we confirm 
the stability results through numerical simulations and we also investigate some global properties of the model and the different kind of disequilibrium dynamics as well as the possibility of coexisting attractors and Arnold tongues structure. Section 5 concludes.

\section{The cobweb model}

Producers form price expectations one period ahead and derive their optimal production decision from expected profit maximisation. Given producers' price forecast $p_{t}^{e}$, profit maximising supply is given by

$$
S\left(p_{t}^{e}\right)=\operatorname{argmax}_{q_{t}}\left\{p_{t}^{e} q_{t}-c\left(q_{t}\right)\right\}=\left(c^{\prime}\right)^{-1}\left(p_{t}^{e}\right)
$$

The cost function $c(\cdot)$ is assumed to be strictly convex so that the second-order condition for profit maximisation is satisfied. The marginal cost function is then invertible and supply is strictly increasing in expected price. In general a strictly convex cost curve leads to a nonlinear, increasing, supply curve. As an example of a nonlinear supply curve, we will consider an increasing convex exponential function of the form, ${ }^{1,2}$

$$
S\left(p_{t}^{e}\right)=e^{b\left(p_{t}^{e}-1\right)} \quad b>0
$$

Consumer demand $D$ depends upon the current market price $p_{t}$. The demand curve can be derived from consumer utility maximisation and throughout the paper we will consider a hyperbolic demand function

$$
D\left(p_{t}\right)=\frac{1}{p_{t}}
$$

Assuming that expectations are homogeneous, i.e. all producers have identical price expectations $p_{t}^{e}$, market clearing implies

$$
D\left(p_{t}\right)=S\left(p_{t}^{e}\right)
$$

yielding the realised market equilibrium price

$$
p_{t}=D^{-1}\left(S\left(p_{t}^{e}\right)\right)
$$

Thus the price dynamics depends on the supply and demand curves, but it also depends crucially on the assumed expectations hypothesis. A different expectations hypothesis would lead to a completely different dynamical system describing price fluctuations.

\section{The role of memory in backward looking expectations}

\subsection{Absence of memory: the case of naive expectations}

The simplest case studied in the 1930s, e.g. by Ezekiel in [9], assumes that producers have naive expectations, that is their prediction correspond to the last observed price $p_{t}^{e}=p_{t-1}$. Under naive expectations the price dynamics reduces to a one-dimensional map

$$
p_{t}=D^{-1}\left(S\left(p_{t-1}\right)\right)=f\left(p_{t-1}\right)
$$


When the demand function is decreasing and the supply is increasing, the map $f=D^{-1} S$ is decreasing. Then the price dynamics has a unique steady state $p^{*}$ where demand and supply intersect.

In the case under study the equilibrium condition reads as

$$
\frac{1}{p_{t}}=e^{b\left(p_{t-1}-1\right)}
$$

which yields the one-dimensional system

$$
p_{t}=\frac{1}{e^{b\left(p_{t-1}-1\right)}}
$$

It is easy to check that the unique steady state is $p^{*}=1$.

According to the well-known cobweb theorem, the local stability of the equilibrium price depends upon the ration of marginal supply and marginal demand at the steady state $p^{*}$. There are two possibilities for the price dynamics:

- if $-1<S^{\prime}\left(p^{*}\right) / D^{\prime}\left(p^{*}\right)<0$ the steady state $p^{*}$ is locally stable and prices converge to it;

- if $S^{\prime}\left(p^{*}\right) / D^{\prime}\left(p^{*}\right)<-1$ the steady state $p^{*}$ is locally unstable and prices diverge from the steady state.

In the case of nonlinear demand and supply, and naive expectations, the steady state stability condition requires

$$
\left|\frac{d p_{t}}{d p_{t-1}}\right|=\left|-b e^{-b\left(p^{*}-1\right)}\right|<1
$$

which is satisfied for $0<b<1$. Thus when the steady state is unstable, prices will converge to a stable 2-cycle, with regular up and down oscillations. Under naive expectations with nonlinear but monotonic demand and supply curves, the dynamics is simple: prices either converge to a stable steady state or prices diverge from the steady converging to a periodic cycle. Figure 1 displays the two possible dynamics, that is convergence to the fundamental price $p^{*}$ (Figure 1(a)) and convergence to a stable 2-cycle (Figure 1(b)).

\subsection{Backward looking expectations with two lags}

In the simple case with only two lags, the memory is introduced in price expectations as:

$$
p_{t}^{e}=(1-w) p_{t-1}+w p_{t-2} \quad w \in[0,1]
$$

i.e. expected price is a weighted average of the two most recent price observations.

Substituting price expectation with memory into the market equilibrium condition, yields

$$
p_{t}=D^{-1} S\left((1-w) p_{t-1}+w p_{t-2}-1\right)
$$


(a)

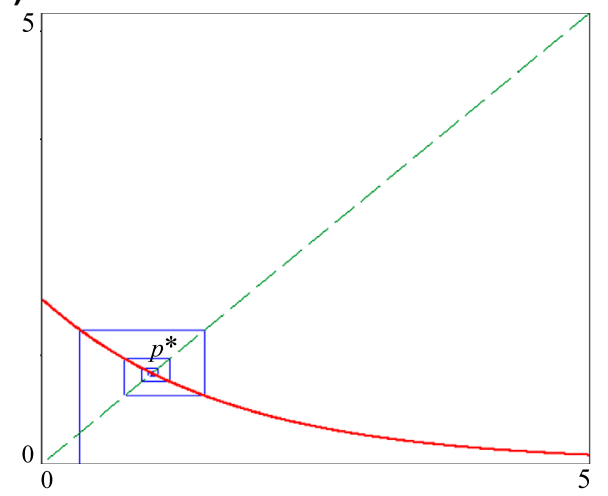

(b)

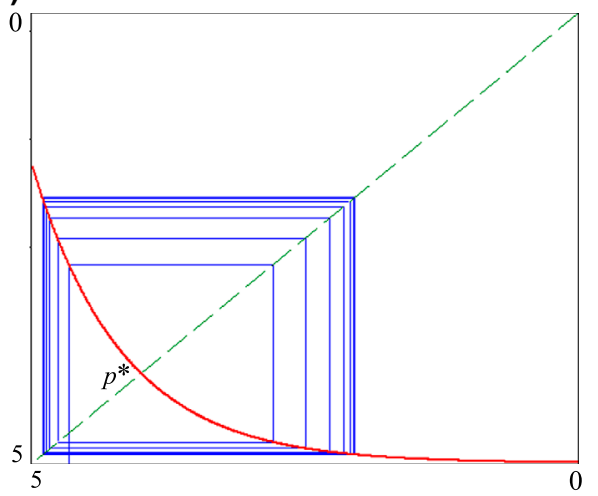

Figure 1. Two different dynamics with nonlinear but monotonic demand and supply curves. (a) convergence to the fundamental price and (b) convergence to a stable 2-cycle.

which is a second order difference equation for the price dynamics and, with supply and demand given by (2)-(3), it specifies to

$$
p_{t}=\frac{1}{e^{b\left((1-w) p_{t-1}+w p_{t-2}-1\right)}}
$$

Writing $p_{t}=x_{t}$ and $p_{t-1}=y_{t}$ an equivalent bi-dimensional map is obtained:

$$
T:\left\{\begin{array}{l}
x_{t+1}=\frac{1}{e^{\left.b(1-w) x_{t}+w y_{t}-1\right)}} \\
y_{t+1}=x_{t}
\end{array}\right.
$$

By imposing the steady state condition $x_{t+1}=x_{t}=y_{t}$ for each $t$, it is easy to check that the map $T$ has a unique steady state $p^{*}=(1,1)$ which does not depend on the memory parameter $w$ and corresponds to the intersection point of demand and supply. The stability properties of the steady state $p^{*}$ are influenced by the parameters $w$ and $b$.

Proposition 1: The steady state $p^{*}=(1,1)$ is locally stable if $b<3$ and $\frac{b-1}{2 b}<w<\frac{1}{b}$

Proof: The Jacobian matrix of map $T$ is given by

$$
J=\left[\begin{array}{cc}
-b(1-w) e^{-b\left[(1-w) x_{t}+w y_{t}-1\right]}-b w e^{-b\left[(1-w) x_{t}+w y_{t}-1\right]} \\
1
\end{array}\right]
$$

and, evaluating at the steady state $p^{*}$, it becomes

$$
J=\left[\begin{array}{cc}
b(w-1) & -b w \\
1 & 0
\end{array}\right]
$$

The set of sufficient conditions for the stability of $p^{*}$ is determined by the usual conditions on the trace $\operatorname{Tr}$ and determinant Det of the Jacobian matrix, namely

$$
\left\{\begin{array}{l}
1+T r+\text { Det }>0 \rightarrow 1+b(1-w)+b w>0 \\
1-T r+D e t>0 \rightarrow 1-b(1-w)+b w>0 \\
\text { Det }<1 \rightarrow b w<1
\end{array}\right.
$$


The first condition is always satisfied being independent on $w$, namely $b>-1$ which is always assumed positive; the second condition can be written as $w>\frac{b-1}{2 b}$ meaning that the steady state loses stability through a period doubling bifurcation at $w^{f}=\frac{b-1}{2 b}$; finally, the third condition yields $w<\frac{1}{b}$ and at $w^{n s}=\frac{1}{b}$ the steady state can be destabilised through a supercritical Neimark-Sacker bifurcation. Simple computations show that if $b<3$ then we obtain

$$
\frac{b-1}{2 b}<w<\frac{1}{b}
$$

and the steady state is locally stable. This concludes the proof.

There are two different ways in which a Neimark-Sacker bifurcation can take place: the supercritical one at which the fixed point $p^{*}$ becomes unstable and an attracting closed curve $\Gamma_{S}$ appears around it, and the subcritical case at which the fixed point $p^{*}$ becomes unstable merging with a repelling closed curve $\Gamma_{U}$, which exists when $E^{*}$ is still stable. In order to study the direction of the appearance of the invariant closed curve when $w^{n s}=\frac{1}{b}$ we follow [10] Theorem 3.5.2 (or [18], Theorem 4.6). At this purpose we consider the set

$$
\Omega=\{(b, w): b>0,0 \leq w \leq 1\}
$$

and we state the following

Proposition 2: If $(b, w) \in \Omega$ with $b \neq\{1,2,3\}$ then at

$$
w=w^{n s}=\frac{1}{b}
$$

the steady state $p^{*}=(1,1)$ undergoes a supercritical Neimark-Sacker bifurcation.

Proof: In Proposition 1 we proved that the steady state $p^{*}$ can be destabilised through a Neimark-Sacker bifurcation. In order to prove that such a bifurcation is of supercritical type, we have to show that

(a) at $w=w^{n s}$ the eigenvalues are such that $\lambda^{n} \neq 1$ when $n=1,2,3,4$;

(b) the transversality condition holds;

(c) a no-degeneracy condition holds.

At $w=w^{n s}$ the eigenvalues are given by

$$
\lambda_{n s}=\frac{1-b+i \sqrt{-b^{2}+2 b+3}}{2}=\cos \vartheta+i \sin \vartheta
$$

and $\bar{\lambda}_{n s}$ where the argument $\vartheta$ is such that $\cos \vartheta=\frac{1-b}{2}$ and $\sin \vartheta=-\frac{(b-3)(1+b)}{2 \sqrt{-b^{2}+2 b+3}}$.

Computing the powers $\lambda_{n s}^{n}=\cos (n \vartheta)+i \sin (n \vartheta), n=1,2,3,4$, we obtain that in the parameter space $\Omega$ they may be equal to 1 if and only if $n=2$ and $b=3, n=3$ and $b=2, n=4$ and $b=1$. Hence point (a) follows by exclusion of the values $1,2,3$ for the parameter $b$.

The transversality condition (b) holds if the derivative of the modulus of the eigenvalues evaluated at the bifurcation value $w^{n s}$ does not vanish. Recalling that $|\lambda|=b w$ we obtain

$$
\frac{d}{d b}\left(\left|\lambda\left(w^{n s}\right)\right|\right)=\frac{1}{b} \neq 0
$$


To prove the no-degeneracy condition (c), we have to show that the Lyapunov coefficient does not vanish. As a first step, to obtain the coefficient we rewrite the map $\mathrm{T}$ in (13) restricted to the centre manifold in a more convenient form, making use of the coordinate change

$$
\left(\begin{array}{l}
x \\
y
\end{array}\right)=\left[\begin{array}{cc}
2 & 0 \\
1-\frac{1}{w} & \frac{\sqrt{-b w^{2}+2 b w+4 w-b}}{\sqrt{b} w}
\end{array}\right]\left(\begin{array}{l}
u \\
v
\end{array}\right)+\left(\begin{array}{l}
1 \\
1
\end{array}\right) .
$$

The new map we obtain by the homeomorphism (15) is topologically conjugated to the map (13) and it is given by

$$
\left(\begin{array}{l}
u^{\prime} \\
v^{\prime}
\end{array}\right)=\left[\begin{array}{cc}
\frac{1-b}{2} & \frac{(b-3)(1+b)}{2 \sqrt{-b^{2}+2 b+3}} \\
-\frac{(b-3)(1+b)}{2 \sqrt{-b^{2}+2 b+3}} & \frac{1-b}{2}
\end{array}\right]\left(\begin{array}{l}
u \\
v
\end{array}\right)+\left(\begin{array}{l}
f(u, v) \\
g(u, v)
\end{array}\right)
$$

where

$$
f(u, v)=-\frac{e^{-b u-\sqrt{-b^{2}+2 b+3} v}\left(e^{b u+\sqrt{-b^{2}+2 b+3} v}-e^{u}\right)}{2}
$$

$g(u, v)$

$$
=\frac{e^{-b u-\sqrt{-b^{2}+2 b+3} v}\left(-e^{u}+b e^{u}+e^{b u+\sqrt{-b^{2}+2 b+3} v}-b e^{b u+\sqrt{-b^{2}+2 b+3} v}+4 u e^{b u+\sqrt{-b^{2}+2 b+3} v}\right)}{2 \sqrt{-b^{2}+2 b+3}}
$$

Following the symbolism used in [10] we obtain

$$
\left.\begin{array}{c}
\xi_{20}=\frac{1}{8}\left[\left(f_{u u}-f_{v v}+2 g_{u v}\right)+i\left(g_{u u}-g_{v v}-2 f_{u v}\right)\right] \\
=\frac{2 i-3 i b^{2}+i b^{3}-2 b \sqrt{-b^{2}+2 b+3}+b^{2} \sqrt{-b^{2}+2 b+3}}{4 \sqrt{-b^{2}+2 b+3}} \\
\xi_{11}=\frac{1}{4}\left[\left(f_{u u}+f_{v v}\right)+i\left(g_{u u}+g_{v v}\right)\right] \\
=\frac{-i+i b+\sqrt{-b^{2}+2 b+3}}{2 \sqrt{-b^{2}+2 b+3}} \\
\xi_{02}=\frac{1}{8}\left[\left(f_{u u}-f_{v v}-2 g_{u v}\right)+i\left(g_{u u}-g_{v v}+2 f_{u v}\right)\right] \\
i-i b+\sqrt{-b^{2}+2 b+3} \\
4 \sqrt{-b^{2}+2 b+3} \\
\xi_{21}=\frac{1}{16}\left[\left(f_{u u u}+f_{u v v}+g_{u u v}+g_{v v v}\right)+i\left(g_{u u u}+g_{u v v}-f_{u u v}-f_{v v v}\right)\right] \\
-i-2 i b+i b^{2}-\sqrt{-b^{2}+2 b+3}+b \sqrt{-b^{2}+2 b+3} \\
4 \sqrt{-b^{2}+2 b+3}
\end{array}\right]
$$




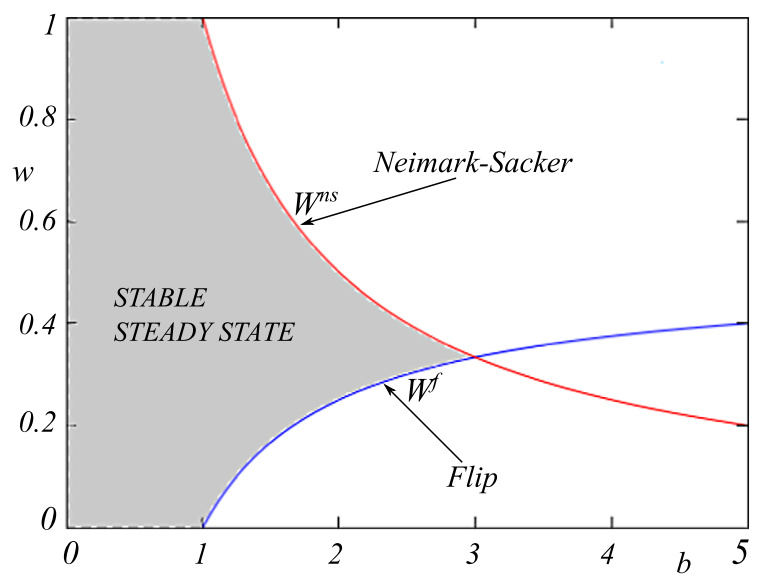

Figure 2. Stability region (grey colour) of the fundamental price $p^{*}$ in the $(b, w)$ parameter space.

Finally, we are able to compute the no-degeneracy parameter

$$
\begin{aligned}
A & =-\operatorname{Re}\left(\frac{\left(1-2 \lambda_{n s}\right) \bar{\lambda}_{n s}^{2}}{1-\lambda_{n s}} \xi_{11} \xi_{20}\right)-\frac{1}{2}\left|\xi_{11}\right|^{2}-\left|\xi_{02}\right|^{2}+\operatorname{Re}\left(\xi_{21} \bar{\lambda}_{N}\right) \\
& =\frac{-b}{4(b+1)}<0
\end{aligned}
$$

Then the parameter $A$ does not vanish and so also condition (c) is satisfied. Since $b>0$ we have $A<0$ and the Neimark-Sacker bifurcation is of supercritical type. This concludes the proof.

From Proposition 2 we also deduce that the parameter value $R_{1: 2}=\left(w^{n s}(3), 3\right)$ corresponds to a 1:2 resonant case, $R_{1: 3}=\left(w^{n s}(2), 2\right)$ corresponds to a 1:3 resonant case and $R_{1: 4}=\left(w^{n s}(1), 1\right)$ corresponds to a 1:4 resonant case. This means that at these parameter values the closed invariant curve might appear in a very peculiar way, or there might be several invariant curves bifurcating from the fixed point. ${ }^{3}$

The stability region of the steady state is depicted in Figure 2 by the grey-shaded area in the parameter plane $(b, w)$ and it is bounded by the curves $W^{f}$ and $W^{n s}$ which respectively denote the flip and and the Neimark-Sacker bifurcation curve.

Along the red curve $W^{n s}$ (Neimark-Sacker bifurcation curve), the product of the eigenvalues of the Jacobian matrix at the steady state equals 1 ; if in addition the eigenvalues are complex, they must lie on the unit circle and so this curve denotes parameter values for which a supercritical Neimark-Sacker bifurcation of the steady state occurs. On the other hand, the blue curve $W^{f}$ (flip bifurcation curve) refers to parameter values for which the Jacobian matrix at the steady state has an eigenvalue equal to -1 (with the other real eigenvalue between -1 and 0 ): hence for these parameters a period-doubling (flip) bifurcation occurs. As it can be seen, along the line $w=0$, at $b=1$ the equilibrium loses stability for increasing values of $b$ through a flip bifurcation. For higher values of the memory parameter $w$ the range of stability of $b$ is increased and it becomes larger and larger for intermediate values of $b$, that is for a more uniform memory distribution. However this is no longer true for high values $w$ : in this case, for increasing values of $b$ the stability of 
(a)

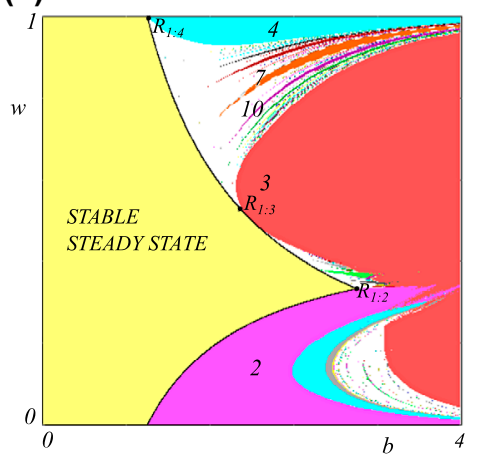

(b)

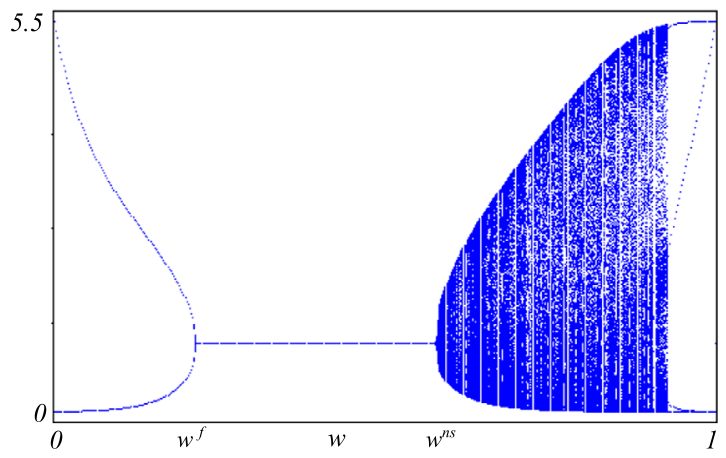

Figure 3. (a) $2 \mathrm{D}$ bifurcation diagram in the $(b, w)$ parameter plane. Regions corresponding to attracting cycles of period $n$ are shown by different colours for different $n$. The black dots refer to the resonance points. (b) Bifurcation diagram, with bifurcation parameter $w$, obtained for $b=1.75$. Starting from the initial condition $x_{0}=1.001$ and $y_{0}=0.4$, for each value of the bifurcation parameter $w$, a trajectory of the model is generated.

the equilibrium is lost through a Neimark-Sacker bifurcation and a quasi-periodic motion around $p^{*}$ is observed. Furthermore, the range of stability of $b$ is reduced for increasing values of $w$.

It needs mentioning that in the cobweb model with naive expectations (no memory) the stability of the steady state requires $0<b<1$ while in the presence of memory two different bifurcation routes to instability may arise, namely the period doubling and the Neimark-Sacker bifurcations of the steady state. Thus, by adding memory in price expectations, there exists a weight distribution such that the steady state is locally stable if the parameter $b$ lies in the region $b \in(0,3)$.

We can state that there exist three different regimes of stability:

(i) if $0<b<1$, the steady state is locally stable, (as for the case of no memory) and by adding memory the system is not destabilised;

(ii) if $1<b<3$, the steady state is locally stable only if the memory parameter $w$ lies in a certain interval, otherwise it is unstable;

(iii) if $b>3$, the steady state is unstable

Let $P_{n}$ denote a region in the $(b, w)$ parameter plane such that for $(b, w) \in P_{n}$ the map $T$ has an attracting cycle of period $n$. We first present a $2 \mathrm{D}$ bifurcation diagram to illustrate the overall bifurcation structure of the parameter space of map $T$ in which each number $n$ corresponds to the different periodicity regions.

Figure 3(a) presents such a bifurcation diagram, where the different periodicity regions, emanating from the Neimark-Sacker bifurcation curve, are shown in different colours (white regions corresponds either to cycles of higher periodicity). This particular structure, also called Arnold tongues or mode-locking tongues, is formed by periodicity regions which are ordered in the parameter space according to the Farey summation rule ${ }^{4}$ applied to the rotation numbers of the related cycles.

It is interesting to observe the qualitative changes in the long-run dynamics when the memory parameter $w$ increases for a fixed value of $b$. Figure 3(b) presents the bifurcation 
(a)

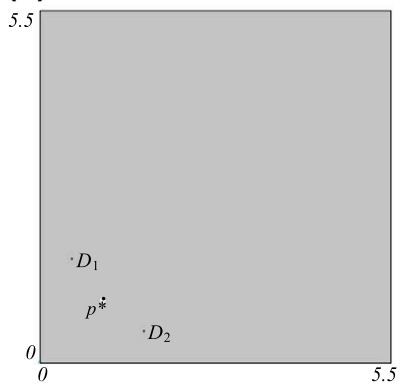

(b)

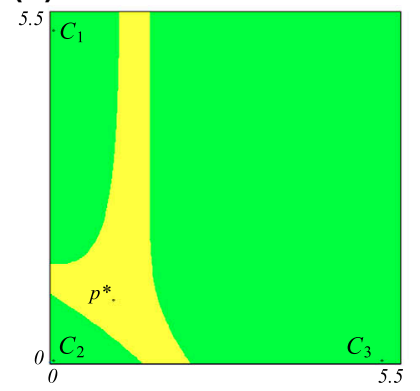

(c)

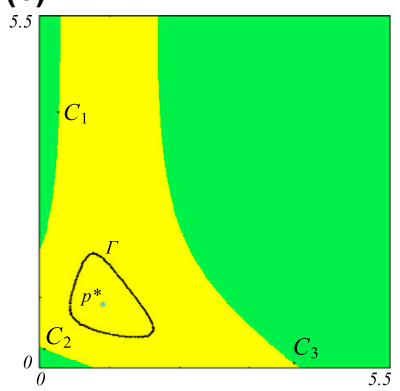

Figure 4. Three representations of the phase plane of the map obtained for $b=1.75$ and different values of $w$. (a) For $w=0.2$ there exists a stable 2-cycle; (b) for $w=0.5$ the stable steady state coexists with a stable 3-cycle; (c) for $w=0.58926$ a closed invariant curve surrounds the unstable steady state and it coexists with a stable 3-cycle.

diagram with respect to the memory parameter $w$, obtained for $b=1.75$. This bifurcation diagram clearly shows the stability range of the equilibrium $p^{*}$ for intermediate value of the memory parameter $w$, as well as the two different kind of local bifurcation through which $p^{*}$ loses stability. This bifurcation diagram confirms the result given in the proposition on local stability and bifurcations of the unique equilibrium.

We observe that when the expectation mechanism gives greater importance to the last realised price, the dynamics is described by a periodic cycle with prices oscillating between high and low values; then, when the parameter $w$ increases, the steady state turns locally stable; finally, as $w$ further grows, meaning that the importance of the last realised price reduces more, the system undergoes a Neimark-Sacker bifurcation at which a closed invariant curve appears. For increasing value of $w$, and in particular for $w>w^{n s}$, the amplitude of the quasi-periodic motion as well as the size of the closed invariant curve increase until it suddenly disappears. This is caused by a global (or contact) bifurcation, as will be shown numerically in the next section. Indeed other interesting dynamic behaviours will be shown by a global numerical exploration that can not be revealed by the only mean of the local stability analysis.

\section{Global analysis: multistability and basins of attraction}

In this section we perform some numerical simulations in order to give a broader view of the global dynamic properties of the model. Indeed the local stability analysis, based on the usual linearization procedure, only yields information about the model's behaviour in a neighbourhood of the steady state $p^{*}$ but it does not give insight into the size and the shape of the basin of attraction of the stable equilibrium. Moreover, for sets of parameters such that the equilibrium price is not stable, we analyse the kind of disequilibrium dynamics that prevails and, even when the equilibrium is locally stable, we show that other attractors exists far from the steady state.

Figure 4 shows the different attractors and their basins of attraction in the phase space $(x, y)$ for the same set of parameters used for the bifurcation diagram of Figure 3 and for different values of the memory parameter $w$ corresponding to three different dynamic scenarios. Figure 4(a), obtained for $w=0.2$ (that is in the case of a high importance give 
(a)

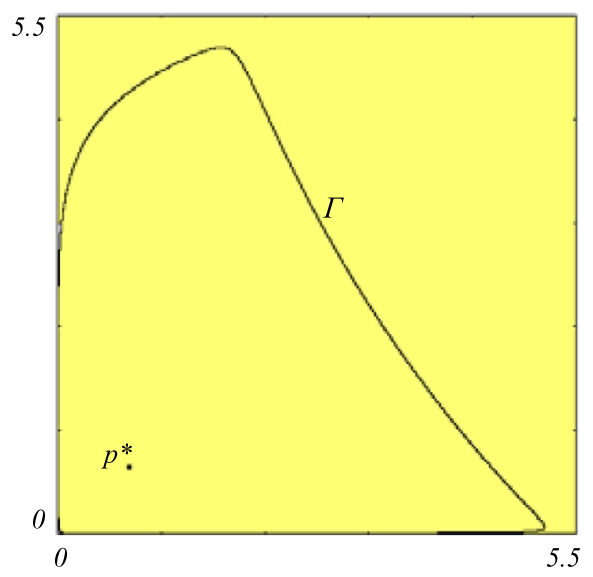

(b)

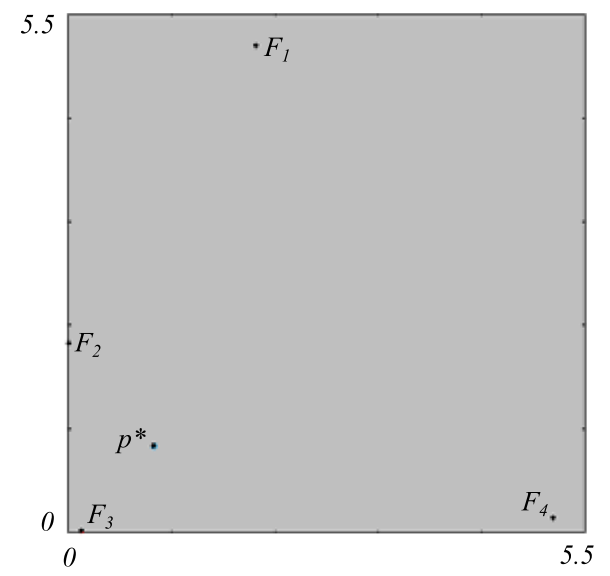

Figure 5. Disappearance of the closed invariant curve and route to periodic oscillations. Phase plane of the map obtained for $b=1.75$ and different values of $w$. (a) As $w$ increases the curve becomes larger and larger $(w=0.9)$, (b) until it breaks and turns into a periodic cycle $(w=0.929)$.

to the last price realisation) shows the basin of attraction (grey colour) of the stable 2-cycle around the unstable equilibrium price $p^{f}$; in Figure 4(b), obtained for $w=0.5$ (that is in the case of equal weight given to the last price realisation and to the price realised one period before), the stable equilibrium point $p^{f}$ coexists with a stable 3-cycle; finally, in Figure 4(c), obtained for $w=0.58926$, a stable closed invariant curve $\Gamma$ surrounds the unstable steady state and it coexists with a stable 3 -cycle. The multistability phenomena observed in Figure 4(c) may be due to the existence of the resonance point $R_{1: 3}$, since the parameter values are very close to it.

As previously stated, the equilibrium price $p^{f}$ is locally stable for intermediate value of the memory parameter; moreover, even if the steady state is locally stable, it can coexist with a periodic attractor. Thus, as the memory parameter $w$ increases, the equilibrium point $p^{f}$ loses stability and a closed invariant curve appears through a supercritical Neimark-Sacker bifurcation. Such a curve coexists with a stable 3-cycle (see Figure 4(c)). After the contact between the points of the cycle of period 3 and the border of its basin of attraction, the 3 -cycle disappears through a saddle-node bifurcation.

Figure 5 illustrates the mechanism leading to the disappearance of the closed invariant curve: as the memory parameter $w$ increases, the curve $\Gamma$, associated to wider and bounded price oscillations, becomes larger and larger (see Figure 5(a)) turning into a cycle of period 4 (see Figure 5(b)): hence, when more weight is given to the price realised two periods before, the price dynamics is expected to be stabilised in the sense that irregular price patterns, such as quasi-periodic cycles, with higher variability may become regular, such as cycles, with lower variability.

The presence of the memory can thus lead to interesting dynamic behaviours. Figure 6(a) presents a 2D bifurcation diagram obtained for different initial conditions with respect to the previous one: from this graph we can notice that the periodicity region corresponding to the attracting 3-cycle intersect the stability region of the equilibrium price $p^{*}$, denoting a situation of coexistence of two attractors. The arrow depicted in such a figure refers to a vertical bifurcation path related to increasing values of the memory parameter $w$, 
(a)

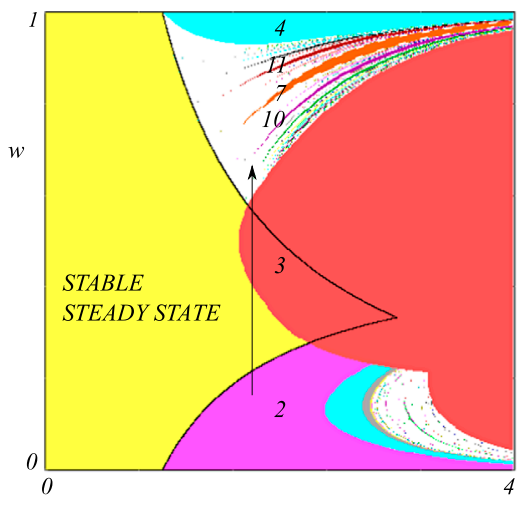

(b)

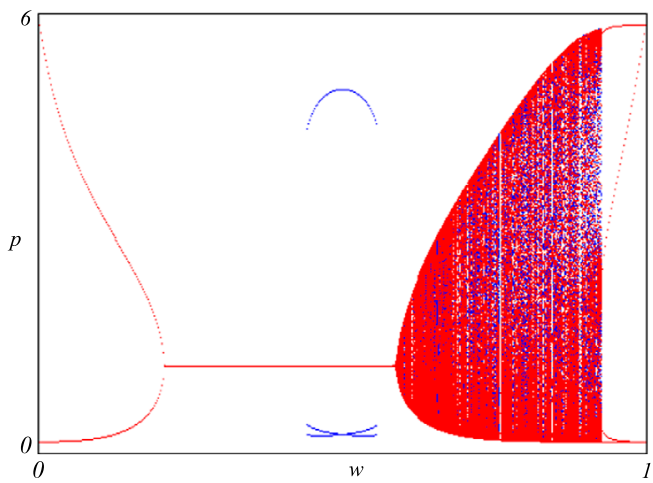

Figure 6. (a) 2D bifurcation diagram in the $(b, w)$ parameter plane. Different colours corresponds to different periodic cycles. The tongue correspondent to the 3-cycle intersects the stability region. (b) bifurcation diagram obtained with $b=1.75$. For each value of the bifurcation parameter $w$, two trajectories of the map are generated, starting from the initial conditions $x_{0}=1.01, y_{0}=0.4$ (red) and $x_{0}=0.4, y_{0}=0.25$ (blue).

being parameter $b$ fixed. To give greater insight to this occurrence, the bifurcation diagram of Figure 6(b) shows the coexistence of the stable steady state and a stable 3-cycle for intermediate values of the memory parameter $w$. Such a bifurcation diagram is obtained by taking, for each value of the bifurcation parameter $w$, two different initial conditions, and the asymptotic portions of the corresponding trajectories are represented in different colours. As can be seen from the figure, in the range $w \in(0.43,0.78)$, i.e. for intermediate values of the memory parameter such that the equilibrium $p^{f}$ is locally stable, a different kind of dynamics can be obtained, given by periodic oscillatory motion, represented by an alternative attractor with its own basin that shares the phase plane with the basin of the locally stable steady state.

\section{Conclusion}

In this paper we have proposed a modification of a nonlinear cobweb model by introducing a memory parameter into the expectation mechanism for the producers: in particular the forecast of future prices has been related to a weighted average of the prices observed in the last two periods. Using a simple nonlinear demand-supply model we have shown that memory can have a qualitatively destabilising effect, beside the commonly acknowledged quantitative stabilisation property. The introduction of memory effects, represented by a convex combination of previous states, has been considered by many authors as a realistic assumption in some economic frameworks (see e.g. [16]). Moreover, as shown in [2], sometimes an increasing memory, that is a larger weight given to the past realisations, has a stabilising effects. This intuition is partially confirmed in the present model in the sense that a configuration of stability can be reached for intermediate values of the memory parameter, that is for a memory distribution close to a uniform average of the two past values of prices. However, we have also shown that the equilibrium may turn locally unstable through a Neimark-Sacker bifurcation. This result is quite intuitive, and it is also observed in the presence of the so-called fading memory involving all the states observed in 
the past (see e.g. [4]). But as stressed in [15], the role of the memory and the time horizons has hardly been studied in the literature, and its role in a general dynamic framework is not a simple matter. Indeed, even in the case of a short memory, dynamics may become complex, as in some ranges of the parameters such that the steady state is locally stable, coexisting periodic attractors and consequent path-dependance behaviour arise.

In fact, when the locally stable steady state coexists with a different kind of attractor, each with its own basin of attraction, a typical situation of corridor stability occurs, as small perturbations around the equilibrium are endogenously recovered by the dynamics of the system, whereas larger perturbations are amplified by the endogenous dynamics, and thus leading to a different disequilibrium dynamics, so that only an external control policy can force the system back to the original equilibrium.

\section{Notes}

1. In general a strictly convex cost curve leads to a nonlinear, increasing, supply curve. Our nonlinear supply curve can, for example, be derived from the convex cost function $c(q)=$ $\frac{q \log q-q}{b}+q+e^{-b}$, where $b$ is a positive constant. See also [14].

2. An exponential supply function like the one here proposed has been estimated in the context of electricity markets and energy prices. See [17] and [22].

3. See [18] for a detailed mathematical analysis about the dynamics around resonance points. See also [1] for a description of the dynamics around a $1: 2$ resonance point in a duopoly model.

4. For example, between two periodicity regions related to cycles with rotation numbers $1 / 2$ and $1 / 3$ there is a region corresponding to cycles with rotation number $2 / 5$.

\section{Acknowledgements}

We thank the Editor in Chief and an anonymous Referee for useful comments and suggestions. The usual caveats apply.

\section{Disclosure statement}

No potential conflict of interest was reported by the authors.

\section{References}

[1] A. Agliari and A. Naimzada, Nonlinear Dynamics of a Cournot Duopoly Game with Differentiated Products, Appl. Math. Comput. forthcoming 2016.

[2] F. Aicardi and S. Invernizzi, Memory effects in discrete dynamical systems, Int. J. Bifurcation Chaos 2(4) (1992), pp. 815-830.

[3] P.L. Boyland, Bifurcations of circle maps: Arnold tongues, bistability and rotation intervals, Commun. Math. Phys. 106(3) (1986), pp. 353-381.

[4] G.I. Bischi and A. Naimzada, Global analysis of a nonlinear model with learning, Econ. Notes 26 (1997), pp. 445-476.

[5] G.I. Bischi and A. Naimzada, A Kaleckian macromodel with memory, in Cycles, Growth and the Great Recession, A. Cristini S.M. Fazzari E. Greenberg and R. Leoni, eds., Routledge, Oxon, Vol. 103, 2014.

[6] J.A. Carlson, An invariably stable cobweb model, Rev. Econ. Stud. 35(3)(1968), pp. 360-362.

[7] C. Chiarella, The cobweb model: Its instability and the onset of chaos, Econ. modell. 5(4) (1988), pp. 377-384. 
[8] R. Dieci and F. Westerhoff, Stability analysis of a cobweb model with market interactions, Appl. Math. Comput. 215(6) (2009), pp. 2011-2023.

[9] M. Ezekiel, The cobweb theorem, Q. J. Econ. 52(2)(1938), pp. 255-280.

[10] J. Guckenheimer and P. Holmes, Nonlinear Oscillations, Dynamical Systems, and Bifurcations of Vector Fields, Springer-Verlag, New York, 1985.

[11] J.M. Holmes and R. Manning, Memory and market stability: The case of the cobweb, Econ. Lett. 28(1) (1988), pp. 1-7.

[12] C.H. Hommes, Adaptive learning and roads to chaos: The case of the cobweb, Econ. Lett. 36(2) (1991), pp. 127-132.

[13] C.H. Hommes, On the consistency of backward looking expectations: The case of the cobweb, J. Econ. Behav. Organiz. 33 (1998), pp. 333-362.

[14] C. Hommes, Behavioral Rationality and Heterogeneous Expectations in Complex Economic Systems, Cambridge University Press, New York, 2013.

[15] C. Hommes, T. Kiseleva, Y. Kuznetsov, and M. Verbic, Is more memory in evolutionary selection (de) stabilizing? Macroecon. Dyn. 16(3) (2012), pp. 335-357.

[16] S. Invernizzi and A. Medio, On lags and chaos in economic dynamic models, J. Math. Econ. 20(6) (1991), pp. 521-550.

[17] T. Kanamura, A supply and demand based volatility model for energy prices, Energy Econ. 31(5) (2009), pp. 736-747.

[18] Y.A. Kuznetsov, Elements of Applied Bifurcation Theory, 3rd ed., Springer-Verlag, New York, 2004.

[19] A. Leijonhufvud, Effective demand failures, Swedish J. Econ. 75(1)(1973), pp. 27-48.

[20] R. Manning, Stability of cobwebs, Econ. Record 46(4) (1970), pp. 588-589.

[21] M. Nerlove, Adaptive expectations and cobweb phenomena, Q. J. Econ. 72(2)(1958), pp. 227240.

[22] R. Takashima, Y. Naito, H. Kimura, and H. Madarame, Investment in electricity markets: Equilibrium price and supply function, 11th Annual Real Options Conference, June, Berkeley, CA, USA, 2007, pp. 6-9. 